DOC,

D101,60/5:

99-03

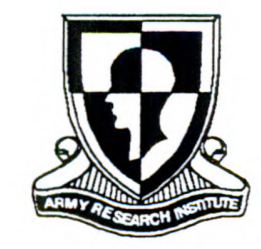

Research Product 99-03

\title{
Development of the COBRAS III Performance Objectives for the Brigade and Battalion Staff Exercise
}

U.S. Army Research Institute for the Behavioral and Social Sciences 